\title{
Identifying occupant presence in a room based on machine learn- ing techniques by measuring indoor air conditions
}

\author{
Lucia Hanfstaengl ${ }^{1, *}$, Michael Parzinger ${ }^{1}$, Uli Spindler ${ }^{1}$, Ulrich $_{\text {Wellisch }}{ }^{1}$, and Markus Wirnsberger ${ }^{1}$ \\ ${ }^{1}$ Rosenheim Technical University of Applied Sciences, Germany
}

\begin{abstract}
Knowing about the presence and number of people in a room can be of interest for precise control of heating, ventilation and air conditioning. To determine the number and presence of occupants cost-effectively, it is of interest to use already existing air condition sensors (temperature, humidity, $\mathrm{CO}_{2}$ ) of the building automation system. Different approaches and methods for determining presence have attracted attention in recent years. We propose an occupancy detection method based on a method of supervised machine learning. In an experiment, measurement data were recorded in a research apartment with controllable boundary conditions. The presence of people was simulated by artificial injection of water vapour, $\mathrm{CO}_{2}$ and heat dissipation. The variation of the number of artificial users, the duration of presence and the supply air volume flow of the ventilation resulted in a total of 720 combinations. By using artificial users, the boundary conditions were accurately defined, and different presence situations could be measured time-effectively. The data is evaluated with a method of supervised machine learning called random forest. The statistical model can determine precisely the number of people in over $93 \%$ of the cases in a disjoint test sample. The experiments took part in the Rosenheim Technical University of Applied Sciences laboratory.
\end{abstract}

\section{Introduction}

\subsection{Scientific Background}

Calculated energy demands are used to compare buildings with each other. Depending on user behaviour and the quality of construction work, actual energy consumption may vary to a greater or lesser extent from the calculated energy demand. The actual energy demand of a building can therefore only be determined by a measurement of the building itself. During monitoring, user behaviour has a strong influence on the result of calculations, so it is of interest to know about the user behaviour in the buildings when making energy evaluations.

Multiple methods for presence detection are in discussion or under investigation. These include movement and presence detectors, user interfaces in the room (light switches, climate control, beamer control, displays, blinds), video surveillance, or even the use of near-field communication to detect the presence of smartphones [1] [2]. Also, different studies look into sound pressure level measurements [3] or chair sensors that detect a person sitting [4]. Not all methods are suitable for obtaining information about the number of people in a room. Some of the sensors may deliver very accurate results. However, data and privacy protection may create problems with user acceptance. In terms of cost efficiency, it would be advantageous to know if and which sensors of a room control system provide reliable data on the presence of people,

\footnotetext{
* Corresponding author: Lucia.Hanfstaengl@th-rosenheim.de
}

as these systems are generally already installed in many rooms.

International research projects such as the IEA Annex 71 [5] have set themselves the goal of improving the prediction, characterisation and quality assurance of the actual energy performance of buildings. Depending on the type of building and the intensity of building automation, the user is one of the most significant influencing factors on the energy demand. Therefore, there is an increasing focus on how to measure user behaviour in situ [1]. Some studies aim to improve building simulation models for predicting energy demand by using more realistic user profiles [6]. Other studies focus on predicting the presence of people to improve the control of heating, ventilation and lighting systems [6], [7], [3], [4], [8]. Further studies see potential to improve facility management, e.g. in room occupancy [6]. Estimation of the presence of people in "real-time" should influence the control of the building technology in such a way that energy can be saved optimally [7]. The presence of people has been examined in offices [6], open-plan offices [9], student apartments [7] and theatres [3].

A frequently used measurement parameter to identify the number of people present is the $\mathrm{CO}_{2}$ concentration [1], [6], [7], [3] because it increases with the number of people. However, the air change, airtightness, wind speed and room volume has also an influence on the increase. Also, air humidity, volatile organic compounds (VOC) and PIR (Passive Infrared Sensor) sensors have been investigated concerning presence predictions and provide results with good model accuracy [7].

In the literature studied here, the presence of people is detected using various statistical analysis methods. Kim 
et al. [6] use three different machine learning methods, which are: classification and regression tree (CART), artificial neural network (ANN) and support vector machine (SVM). Jiang et al. [9] work with the Scaled Extreme Learning Machine (FS-ELM) algorithm feature. In Pedersen et al. [7], they apply a series of decision rules (binary) to the curve of sensor data. Zuraimi et al. [3] chose three standard machine learning methods: 1) ANN, 2) Prediction Error Minimization (PEM) and 3) SVM, as these are widely used and easy to implement. Ryu et al. [8] apply a decision tree algorithm in the first step, and in the second step, select a model for predicting presence using a hidden Markov model (HMM).

\subsection{Contribution}

This thesis considers the identification of the presence of people from the experimental design through to the statistical analysis. The study accounts the interaction between and the effectiveness of the methods employed. The aim is to improve the test design for further investigations continuously and to prepare it for applications in the field.

This study explores ways of using random forest to determine the presence of people and the number of persons that are present. The method of analysis used is random forest, a method of supervised machine and statistical learning [10]. This method is an ensemble method in which bootstrapping (bagging) combined with a random selection of features are used to create multiple, uncorrelated decision trees. This nonparametric and nonlinear method offers high flexibility, e.g. it does not require a distribution assumption or a detailed physical model. The method can deal with classification and regression tasks. This thesis is a multicategorical classification task. The method is very well suited for parallelization of the calculation steps, which makes it possible to evaluate even large amounts of data quickly.

Furthermore, this study investigates which and how many sensors of a room control system are needed for the presence prediction. A range of different room sensors is included in the analysis so that it is possible to analyse which parameters are decisive and whether the prediction accuracy increases with observations of different sensors. With the help of importance measures, the method random forest offers the possibility of creating a ranking list of features (i.e. influencing variables based on the sensor values or measurement parameters) concerning their prediction quality. Also, based on the importance measures, faulty sensors can be detected by the low importance assigned to them.

\section{Methods}

\subsection{Experimental design}

This study estimates the presence of people using a method of machine learning, random forest. The data required for the development of the statistical models was generated in a series of experiments. In a first experiment at the Rosenheim Technical University of Applied Sciences laboratory, methods to predict the presence of people were developed using data of a test series with real human subjects. A second test series measured human impact represented by artificial users at a test apartment of Rosenheim Technical University of Applied Sciences laboratory. The apartment is located in a warehouse, so there is no impact from external influences like wind or solar radiation. The apartment has a floor area of $70 \mathrm{~m}^{2}$. The measurement took place in the sleeping room, with the dimensions $5.7 \mathrm{~m} \cdot 3 \mathrm{~m} \cdot 2.8 \mathrm{~m}$ $(L \cdot W \cdot H)$. The apartment has a central ventilation system with a heat recovering unit so that the temperature fluctuations of the supply air are very low. The room used for the measurements has a supply air vent through which the air enters the room. The air leaves the room through a gap under the door.

Sensirion SCD30 sensors measured temperature, humidity and $\mathrm{CO}_{2}$ concentration at four points in the room and one point in the supply air. The Sensirion SCD30 sensor is a common sensor for HVAC applications. It can measure temperature at $30^{\circ} \mathrm{C}$ with an accuracy of $\pm 0.41^{\circ} \mathrm{C}$, relative humidity with $\pm 3 \%$ and $\mathrm{CO}_{2} \pm(30 \mathrm{ppm}+3 \% \mathrm{MV})$. The used measurement interval was 30 seconds.

Artificial users generated the human impact on the indoor air condition of the room. Cooling load simulators described in DIN EN 14240 [11] provided the thermal heat. According to VDI 2078 [12], the sensitive heat dissipation of a person that performs office work at $20^{\circ} \mathrm{C}$, is $90 \mathrm{~W}$. Light bulbs inside the dummies generated the heat.

$\mathrm{CO}_{2}$ was released into the room air at the height of $1.0 \mathrm{~m}$ in the middle of the room. The $\mathrm{CO}_{2}$ was stored in a bottle outside the apartment and transported via a hose to the test room. The $\mathrm{CO}_{2}$ emission per person for office work is $22 \mathrm{l} / \mathrm{h}$. Water was vaporized in the middle of the room by an ultrasonic nebulizer to simulate moisture emission of persons. A person has a moisture emission of $50 \mathrm{~g} / \mathrm{h}$ at the given boundary conditions. Figure 1 shows the layout of the test room and the positions of the components.

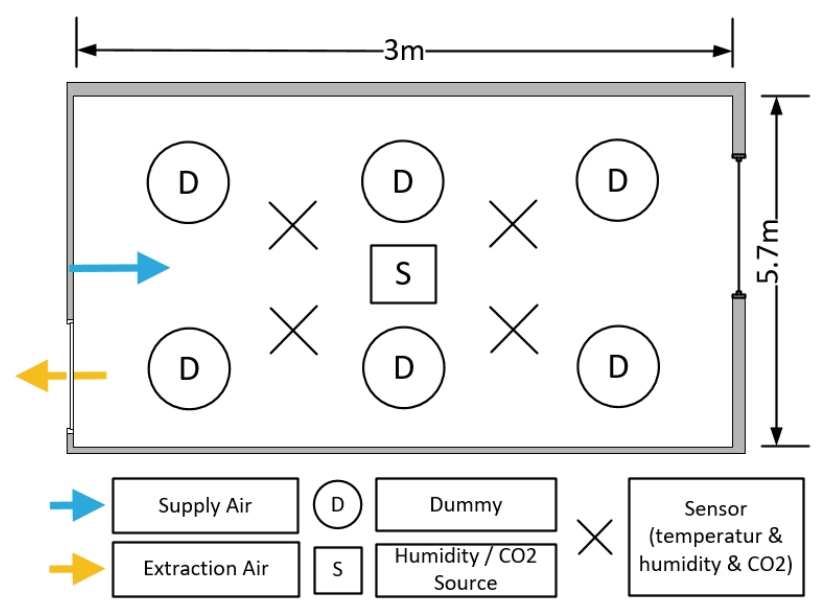

Fig. 1. Experimental setup in the research apartment.

The number of persons present varied from zero to five, the presence time from 10 to 60 minutes with a timestep of 10 minutes. An artificial user control automatically switched 


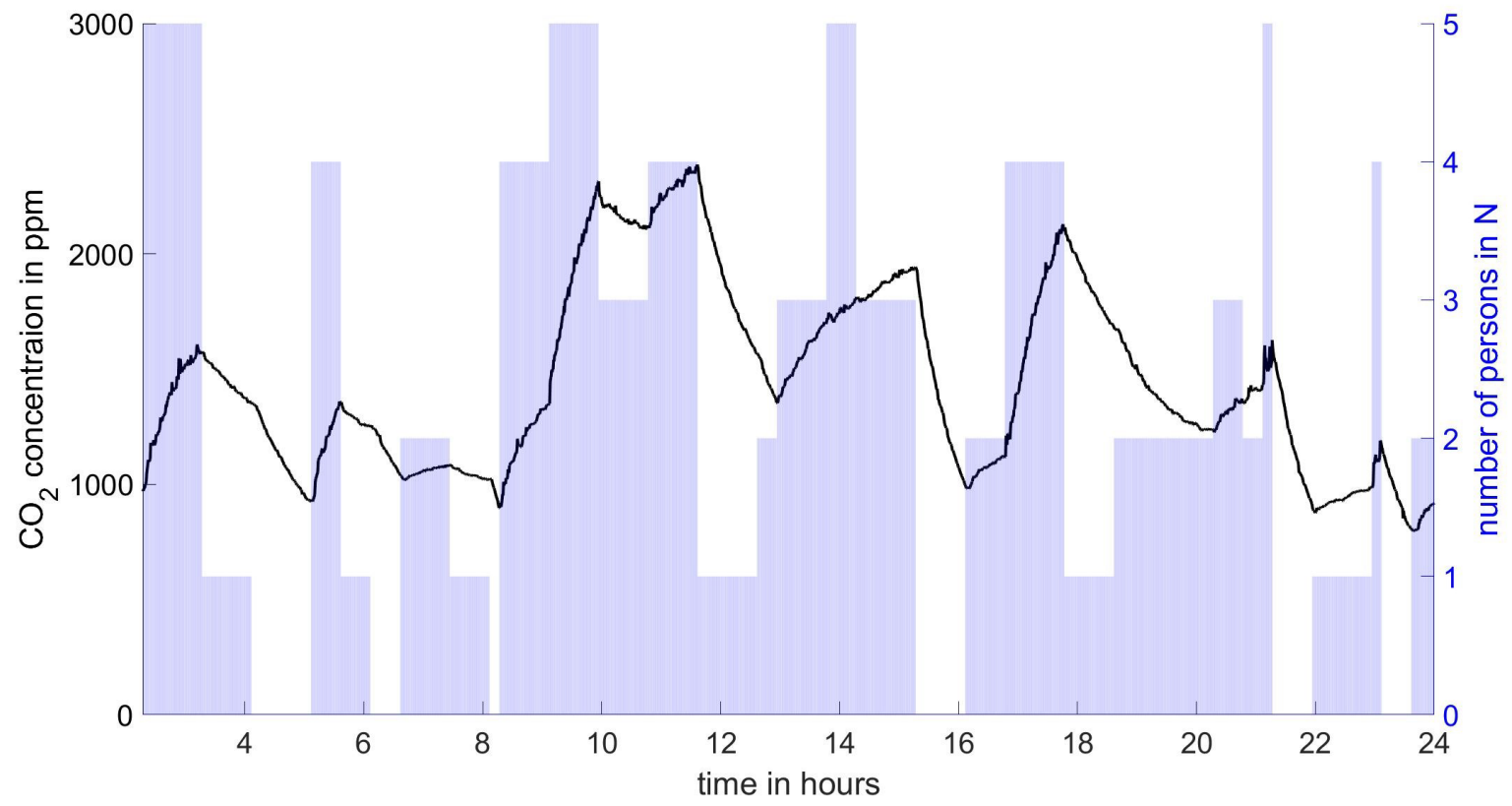

Fig. 2. $\mathrm{CO}_{2}$ concentration and number of persons for the first day of the measurement campaign.

the number of persons present. Thermal heat, $\mathrm{CO}_{2}$ and humidity emission were regulated according to the number of persons present. Additionally, the supply air volume flow was changed between low and high (30 and $90 \mathrm{~m}^{3} / h$ ). For the test design, all variables were randomized with 20 repetitions of each variable set resulting in an experiment with a total of 720 settings. Table 1 shows the frequencies of person count and presence time in the experimental design.

Table 1. Design of experiments.

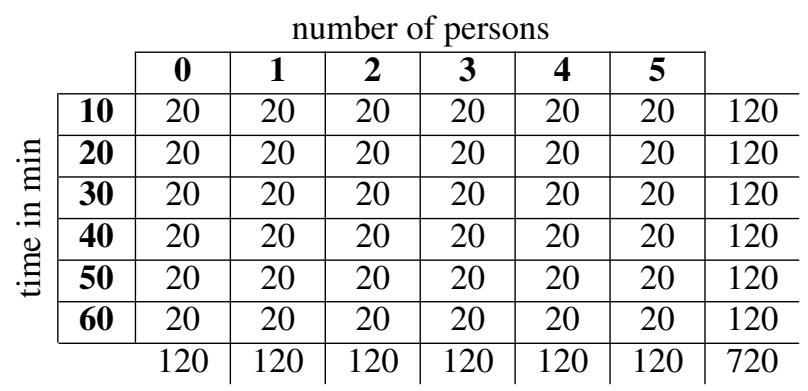

\section{Results}

\subsection{Results from experimental study}

Figure 2 shows exemplary the $\mathrm{CO}_{2}$ concentration during the first measurement day. The height of the bars indicates the number of people present. As is would be expected, the slope of the $\mathrm{CO}_{2}$ concentration curve gets greater with an increasing number of persons and lower with decreasing person numbers.

The relative humidity increases with increasing absolute humidity and decreases with increasing temperature.
Since both absolute humidity and temperature increase when a person enters a room, the correlation between the number of persons and the relative humidity is weak. For that reason, the absolute humidity was calculated. The reason for the average temperature in the room of $30^{\circ} \mathrm{C}$ is the heat recovery of the ventilation system and that the experiments took place in summer.

First of all, the data is exploratory analysed for the suitability of the parameters to determine or to predict the number of persons. The changes of parameters over time were calculated for each presence period and normalised to the change in one hour, shown in Figure 3. There is a correlation between the absolute humidity and the number of people. The median value of the change in absolute humidity for one person is $-1.7 \mathrm{~g} / \mathrm{h}$. The medians rise steadily with an increasing number of persons to a value of $+1.9 \mathrm{~g} / \mathrm{h}$ for five persons present. Such a significant correlation could not be established for the temperature change. The medians vary between -0.3 and $+0.3 \mathrm{~K} / \mathrm{h}$. There is a correlation between the number of persons and $\mathrm{CO}_{2}$. The median for the change of $\mathrm{CO}_{2}$ for one person is $-847 \mathrm{ppm} / \mathrm{h}$. The median rises steadily as the number of person increases and is at a value of $+1010 \mathrm{ppm} / \mathrm{h}$ for five persons present. The exploratory analysis shows that absolute humidity and $\mathrm{CO}_{2}$ are better suited to determine the number of people than temperature.

\subsection{Results of the statistical model}

In this study, the machine learning method random forest predicts the number of persons present. The method uses all the sensor values as possible features. For each sensor the difference between the current value and the values $30 \cdot d$ seconds ago, where $d=1,2, \ldots, 10$, are added as additional features. To evaluate the final models the data get split up 

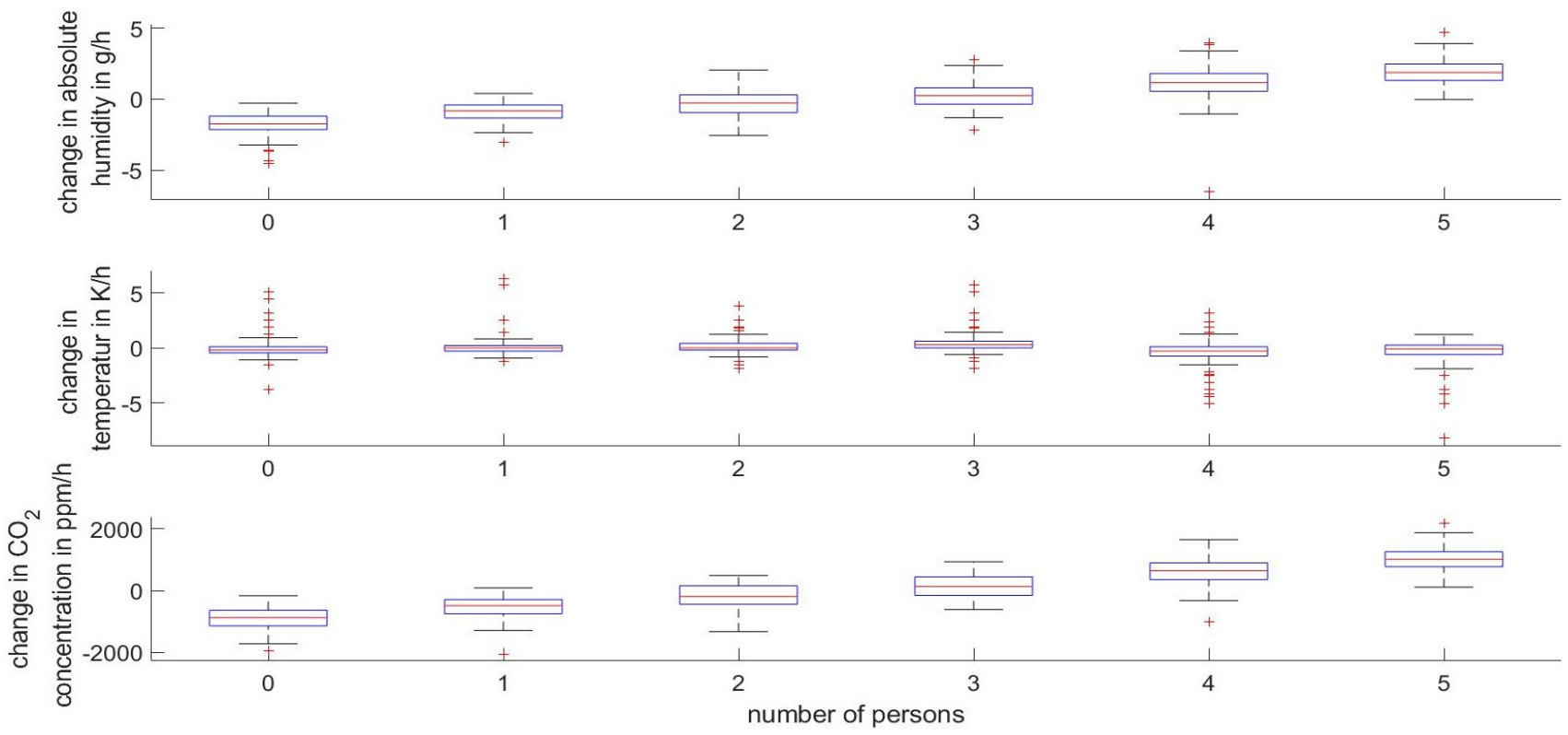

Fig. 3. Box plots of change of the parameters for all presence times in dependency of the number of persons.

into a training and a test set, this means that $75 \%$ of the data is randomly used for the training and $25 \%$ is used as disjoint test data. The training data is used to specify and compute the model, while the test data is only used at the end of the model generating process to see how well the model performs. The relative proportion for the number of persons should be similar in each data set.

\subsubsection{Performance of the model}

To find the best model, a measure to distinguish between suitable and unsuitable models is needed. One possibility is to use the confusion matrix. At first, the train data is again randomly split up into $2 / 3$ train data and $1 / 3$ validation data. Then the models computed on the training data are used to predict the responses of the validation data. The confusion matrix of table 2 is obtained. In table 2 the highest values

Table 2. Confusion matrix on the validation data. The element in row $i$ and column $j$ shows how often $i$ people are predicted while $j$ people are actually present.

\begin{tabular}{c|cccccc}
\multicolumn{7}{c}{ Observation $y$} \\
Prediction $\hat{y}$ & 0 & 1 & 2 & 3 & 4 & 5 \\
\hline 0 & 1874 & 42 & 11 & 5 & 10 & 4 \\
1 & 44 & 2058 & 62 & 15 & 7 & 7 \\
2 & 17 & 51 & 1748 & 64 & 19 & 11 \\
3 & 19 & 20 & 59 & 1789 & 60 & 23 \\
4 & 6 & 7 & 13 & 49 & 1621 & 110 \\
5 & 16 & 7 & 17 & 14 & 99 & 1716
\end{tabular}

are on the diagonal. This means that in the most cases the prediction is correct. A problem with confusion matrices is that it is not easy to compare two of them. Therefore a loss matrix is defined as $\left(l_{i, j}\right)_{i, j=0, \ldots, 5}$ with $l_{i, j} \geq 0$ if $i \neq j$ and $l_{i, j}=0$ if $i=j$ for $i, j=0, \ldots, 5$. The value $l_{i, j}$ is the loss when $i$ people are predicted but $j$ people are observed. Let the confusion matrix be defined as $\left(c_{i, j}\right)_{i, j=0, \ldots, 5}$ with $c_{i, j}$ the number of predictions for $i$ persons under the condition that $j$ persons were present. By that the (relative) loss is defined as

$$
\text { Loss }=\frac{1}{n} \sum_{i, j=0}^{5} c_{i, j} l_{i, j}
$$

with $n=\sum_{i, j=0}^{5} c_{i, j}$ the total number of observations. Three different loss matrices are considered. A first example of a loss matrix is defined in table 3 . In this matrix each missclassification has the same loss. In this study, a prediction

Table 3. Binary loss matrix. Each miss-classification has the same loss.

\begin{tabular}{c|cccccc}
\multicolumn{7}{c}{ Observation $y$} \\
Prediction $\hat{y}$ & 0 & 1 & 2 & 3 & 4 & 5 \\
\hline 0 & 0 & 1 & 1 & 1 & 1 & 1 \\
1 & 1 & 0 & 1 & 1 & 1 & 1 \\
2 & 1 & 1 & 0 & 1 & 1 & 1 \\
3 & 1 & 1 & 1 & 0 & 1 & 1 \\
4 & 1 & 1 & 1 & 1 & 0 & 1 \\
5 & 1 & 1 & 1 & 1 & 1 & 0
\end{tabular}

of four people while five people actually are in the room is not as far away from the truth as a classification of zero people. As a result the absolute loss matrix table 4 and the quadratic loss matrix table 5 are defined. These matrices are now used to calculate the loss from the confusion matrix in table 2 . The results are shown in the table 6 .

\subsubsection{Tuning of the model}

The method avoids overfitting if the number of features, there are a total of 176 possible features, is reduced. For this purpose, the random forest method contains a kind of an importance list with respect to the predictive power of the features. It sorts the features from the most important 
Table 4. Absolute loss matrix. The loss is the absolute distance between the observed value and the predicted value.

\begin{tabular}{c|cccccc}
\multicolumn{7}{c}{ Observation $y$} \\
Prediction $\hat{y}$ & 0 & 1 & 2 & 3 & 4 & 5 \\
\hline 0 & 0 & 1 & 2 & 3 & 4 & 5 \\
1 & 1 & 0 & 1 & 2 & 3 & 4 \\
2 & 2 & 1 & 0 & 1 & 2 & 3 \\
3 & 3 & 2 & 1 & 0 & 1 & 2 \\
4 & 4 & 3 & 2 & 1 & 0 & 1 \\
5 & 5 & 4 & 3 & 2 & 1 & 0
\end{tabular}

Table 5. Quadaratic loss matrix. The loss is the quadratic distance between the observed value and the predicted value.

\begin{tabular}{c|cccccc}
\multicolumn{7}{c}{ Observation $y$} \\
Prediction $\hat{y}$ & 0 & 1 & 2 & 3 & 4 & 5 \\
\hline 0 & 0 & 1 & 4 & 9 & 16 & 25 \\
1 & 1 & 0 & 1 & 4 & 9 & 16 \\
2 & 4 & 1 & 0 & 1 & 4 & 9 \\
3 & 9 & 4 & 1 & 0 & 1 & 4 \\
4 & 16 & 9 & 4 & 1 & 0 & 1 \\
5 & 25 & 16 & 9 & 4 & 1 & 0
\end{tabular}

Table 6. Loss of the confusion matrix in table 2 in relation to three different loss matrices.

\begin{tabular}{c|c} 
Loss-Matrix & multi-dataset \\
\hline Binary & 0.076 \\
Absolute & 0.113 \\
Quadratic & 0.234
\end{tabular}

to the least important feature and calculates the importance with an important measure. The $\mathrm{R}$ package ranger [13] uses three different types of important measures, named "permutation", "impurity" and "impurity corrected".

Another approach for an optimised result is to change the settings of the hyperparameters in the random forest. In this study, the main focus is on the hyperparameter mtry. It is relevant to know that a random forest is a combination of several decision trees. A decision tree consists of knots. The method randomly draws a series of features and determines the best distribution for a node. The number of features drawn for each node is specified by the mtry parameter, see Breiman [10] for more information. It is of interest to find an optimal model by analysing the combinations of importance measure, number of features and mtry. Thus, the random search method is used to randomly try out some combinations and see which of them fit best. The followings steps are performed:

1. Importance lists with the measures permutation, impurity and impurity-corrected are calculated.

2. Draw an importance measure at random.

3. Draw the number of features at random and define this number as $p$.

4. Draw a random value for mtry between 1 and $p$.
5. Create a random forest with the best features $p$ according to the drawn importance measure and with the drawn value for mtry.

6. Use the validation data to create a confusion matrix and save this matrix in a list.

\section{Repeat steps 2-6.}

Figure 4 shows the results of those steps. The loss increases when the method uses less than 50 features. It seems that low values for mtry work better than high values, and that the impact of the importance list is small. The information leads to a repetition of the random search on a smaller area and results in two optimal random forest models. One optimised model with 120 features and with a mtry value of 30 and second a model with 50 features and the mtry value of 6 . It can be assumed that the model with 120 features predicts the presence of people more accurate, but if a small model is preferred the model with 50 features is recommended. The 25 most important features are listed in figure 5.

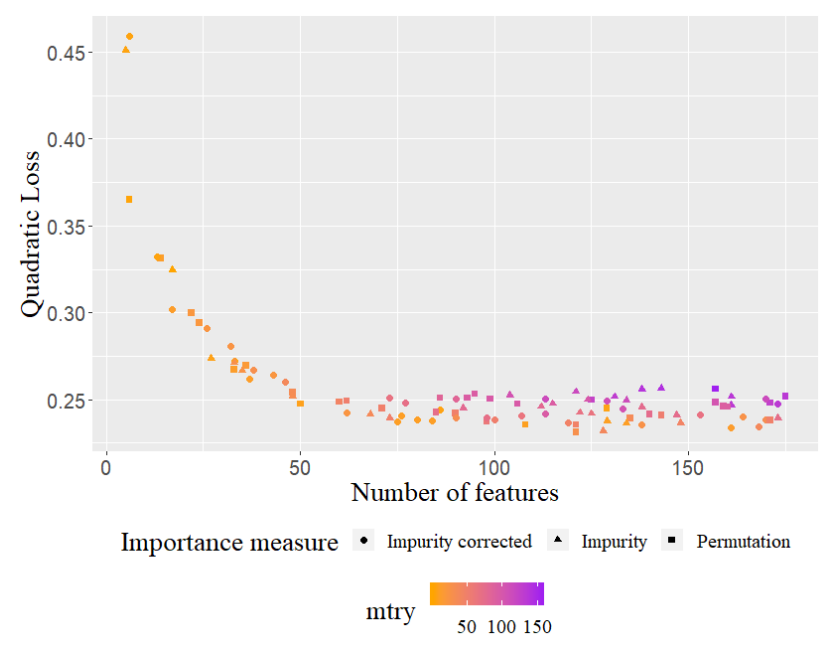

Fig. 4. Result of the random search. The $x$-axis shows the number of features the $y$-axis the loss calculated with the quadratic loss matrix in table 5. The colour shows the value of mtry and the symbol shows the importance list that was used to find the best features.

\subsubsection{Evaluation of the model}

The evaluation of the models uses the complete training and validation sets. The remaining $25 \%$ test data were not taken into account during model creation and model selection. The test data are used to evaluate the selected models afterwards. The confusion matrices are shown in the following tables 7 and 8 . 


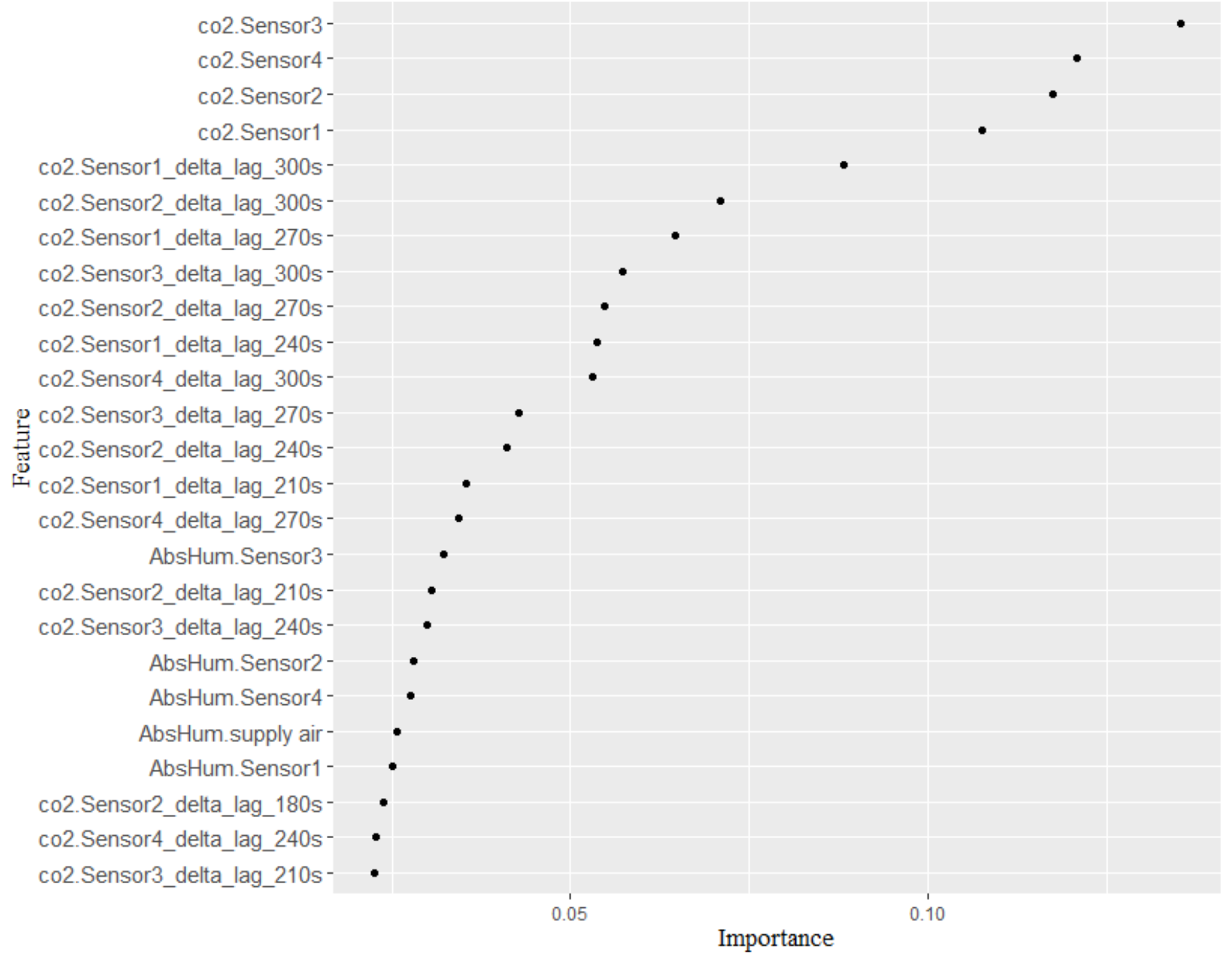

Fig. 5. List of the 25 most important features calculated with the permutation measure. The description 'co2.Sensor' stands for $\mathrm{CO}_{2}$ concentration measurement and the 'AbsHum.Sensor' for absolute humidity measurement. The time lag 'delta_lag_30 $\cdot d \mathrm{~s}$ ' is the difference between the current value and the values $30 \cdot d$ seconds ago.

Table 7. Confusion matrix on the test data. Model uses 120 features and a mtry value of 30. The element in row $i$ and column $j$ shows the number of $i$ people that are predicted while $j$ people were present.

\begin{tabular}{c|cccccc}
\multicolumn{7}{c}{ Observation $y$} \\
Prediction $\hat{y}$ & 0 & 1 & 2 & 3 & 4 & 5 \\
\hline 0 & 1852 & 46 & 13 & 3 & 4 & 4 \\
1 & 25 & 1974 & 47 & 16 & 9 & 7 \\
2 & 9 & 34 & 1723 & 56 & 17 & 10 \\
3 & 14 & 26 & 42 & 1809 & 67 & 32 \\
4 & 14 & 10 & 18 & 58 & 1721 & 111 \\
5 & 12 & 3 & 13 & 22 & 93 & 1780
\end{tabular}

Table 9 lists the results of the losses. The model with 120 features shows slightly better results than the model with 50 features. But the loss differences between 0.004 and 0.007 are quite small and could also be random. The binary loss in table 9 indicates that the models predict in over $92 \%$ of the time the correct number of people.
Table 8. Confusion matrix on the test data. Model uses 50 features and a mtry value of 6 . The element in row $i$ column $j$ shows the number of $i$ people that are predicted while $j$ people were present.

\begin{tabular}{c|cccccc}
\multicolumn{7}{c}{ Observation $y$} \\
Prediction $\hat{y}$ & 0 & 1 & 2 & 3 & 4 & 5 \\
\hline 0 & 1850 & 63 & 15 & 4 & 3 & 4 \\
1 & 24 & 1956 & 44 & 15 & 12 & 7 \\
2 & 9 & 36 & 1724 & 45 & 18 & 10 \\
3 & 20 & 26 & 40 & 1824 & 80 & 34 \\
4 & 11 & 9 & 16 & 57 & 1693 & 120 \\
5 & 12 & 3 & 17 & 19 & 105 & 1769
\end{tabular}

Table 9. Losses of the confusion matrix in table 7 and 8 in relation to three different loss matrices.

\begin{tabular}{c|cc} 
Loss-Matrix & 120 features & 50 features \\
\hline Binary & 0.071 & 0.075 \\
Absolute & 0.107 & 0.112 \\
Quadratic & 0.220 & 0.227
\end{tabular}




\subsection{Analysis of prediction errors}

A detailed analysis of the prediction errors shows the strengths of the method. Of particular interest are the following two questions: 'Do the errors occur more frequently after a change in the number of persons or during a period of attendance?' and 'Is a change from zero to one person more difficult for the model to determine than a change from four to five persons?'

Figure 6 shows the ratio of false predictions to overall predictions for a specific period (e.g. $0.0-5.0 \mathrm{~min}$ ) as a function of the length of the present time and the time since the number of persons changed. The data show that there are no significant time points at which errors occur more frequently. The errors of all combinations of changes are

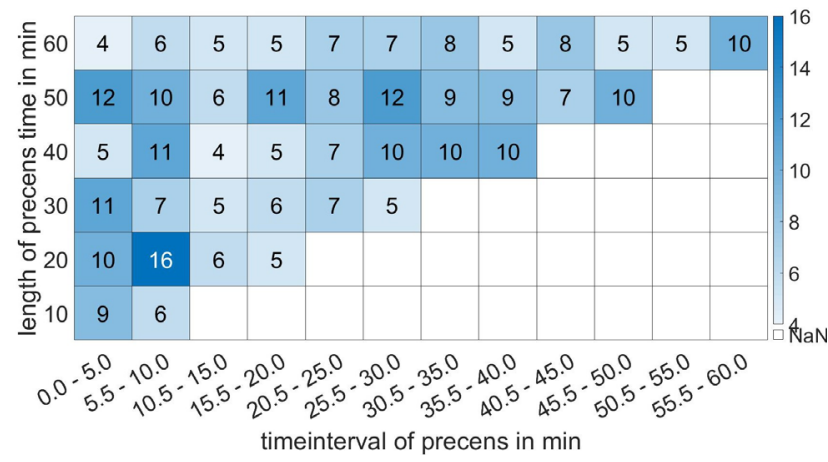

Fig. 6. Ratio of false predictions to overall predictions as a function of the length of the presence time and the time since the number of persons has changed.

calculated to find out whether switching between a certain number of people causes more failures than other sources of error. Figure 7 shows the relative error, that is the number of false predictions in relation to the number of total predictions of a specific combination. The y-axis presents the number of persons of the previous presence phase, and the $\mathrm{x}$-axis presents the number of persons of the current presence phase. The analysis shows no significantly higher error rate for a particular group of combinations neither for small changes nor great changes in the number of people present.

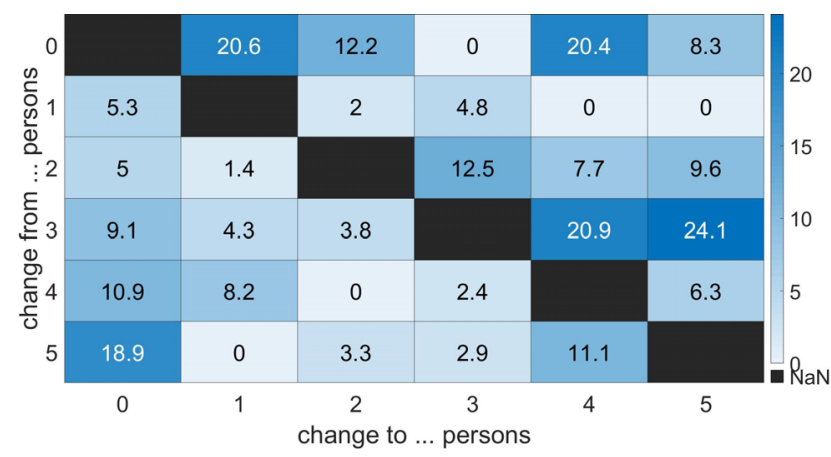

Fig. 7. Ratio of false predictions to overall predictions in the first $300 \mathrm{sec}$ of a presence phase, depending on the number of persons of the previous and the actual presence phase.

\section{Conclusions}

This paper studied the possibilities of determining the number of people in a room based on humidity, temperature and $\mathrm{CO}_{2}$. A method of statistical/machine learning called random forest predicted the number of people present. An experiment with artificial users generated the data to train and validate the model. A random search was applied to tune and select an optimal model. The analysis assessed the data using different valuation methods. Based on a binary loss matrix, the selected model was able to determine on test data, the number of people between 0 - 5 with high accuracy of $93 \%$. The importance measure reflects the importance of particular parameters for the model. The most important features were the current $\mathrm{CO}_{2}$ measurements, as well as the difference between the current $\mathrm{CO}_{2}$ value and the value some time ago.

Future investigations should concern about the predictive potential of each measured variable as the only feature used in a single feature model. For this, the analysis compares models which use only $\mathrm{CO}_{2^{-}}$, temperature-, or humidity-values. For the current investigation, the parameters were measured at multiple points in the room. The influence of the number of measurement points on the prediction accuracy is part of future research. Considering the time lagged features, the most important ones are those with the largest time differences. Therefore it could be useful to create time lag features with even higher time differences. The model optimisation part so far used the hyperparameter mtry only. The other hyperparameters of a random forest could also be optimised by a random search or with other approaches. For the evaluation, the question arises if the performed random split in training and test data is the most meaningful way to estimate the accuracy of the final model. Due to the time series structure of the data, it is likely to obtain other results if, for example, the first $75 \%$ of the data are used for training and the last $25 \%$ are used as test data. This approach would also make it possible to examine whether a subsequent adjustment of the forecasts by estimating the trend could improve them.

Supported by: Federal Ministry for Economic Affairs and Energy on the basis of a decision by the German Bundestag. Projectnumber FKZ03ET1509C.

\section{References}

[1] D. Yan, W. OBrien, T. Hong, X. Feng, H.B. Gunay, F. Tahmasebi, A. Mahdavi, Occupant behavior modeling for building performance simulation: Current state and future challenges, Energy and Buildings, Vol. 107, pp. 264 - 278 (2015)

[2] L. Tianzhen Hong, Technical report: Studying occupant behavior in buildings: Methods and challenges, International Energy Agency, EBC Annex 66 (2017), https://annex66.org/?q=Publication

[3] M. Zuraimi, A. Pantazaras, K. Chaturvedi, J. Yang, K. Tham, S. Lee, Predicting occupancy counts using physical and statistical co2-based modeling method- 
ologies, Building and Environment, Vol. 123, pp. 517 - 528 (2017)

[4] T. Labeodan, W. Zeiler, G. Boxem, Y. Zhao, Occupancy measurement in commercial office buildings for demand-driven control applicationsa survey and detection system evaluation, Energy and Buildings, Vol. 93, pp. 303 - 314 (2015)

[5] IEA ECB Annex 71. Building Energy Performance Assessment Based on In-situ Measurements, Website (2016-2021), https://www.ecbcs.org/ projects/project?AnnexID=71

[6] S. Kim, Y. Song, Y. Sung, D. Seo, Development of a consecutive occupancy estimation framework for improving the energy demand prediction performance of building energy modeling tools, Energies, Vol. 12, 433. (2019)

[7] T.H. Pedersen, K.U. Nielsen, S. Petersen, Method for room occupancy detection based on trajectory of indoor climate sensor data, Building and Environment,
Vol. 115, pp. 147 - 156 (2017)

[8] S.H. Ryu, H.J. Moon, Development of an occupancy prediction model using indoor environmental data based on machine learning techniques, Building and Environment, Vol. 107, pp. 1 - 9 (2016)

[9] C. Jiang, M.K. Masood, Y.C. Soh, H. Li, Indoor occupancy estimation from carbon dioxide concentration, Energy and Buildings, Vol. 131, pp. 132 - 141 (2016)

[10] L. Breiman, Random forests, Machine Learning, Vol. 45, pp. 5-32 (2001)

[11] EN 14240:2004-04 Ventilation for buildings - Chilled ceilings - Testing and rating (2004)

[12] VDI 2078:2015-06 Calculation of thermal loads and room temperatures (design cooling load and annual simulation) (2004)

[13] M.N. Wright, A. Ziegler, ranger: A fast implementation of random forests for high dimensional data in $C++$ and $R$, Journal of Statistical Software, Vol. 77, pp. 1-17 (2017) 